\title{
Trading wind power through physically settled options and short-term electricity
} markets

\author{
Papakonstantinou, Athanasios; Champeri, Georgia; Delikaraoglou, Stefanos; Pinson, Pierre
}

Published in:

Wind Energy

Link to article, DOI:

10.1002/we.2383

Publication date:

2019

Document Version

Peer reviewed version

Link back to DTU Orbit

Citation (APA):

Papakonstantinou, A., Champeri, G., Delikaraoglou, S., \& Pinson, P. (2019). Trading wind power through physically settled options and short-term electricity markets. Wind Energy, 22(11), 1487-1499.

https://doi.org/10.1002/we.2383

\section{General rights}

Copyright and moral rights for the publications made accessible in the public portal are retained by the authors and/or other copyright owners and it is a condition of accessing publications that users recognise and abide by the legal requirements associated with these rights.

- Users may download and print one copy of any publication from the public portal for the purpose of private study or research.

- You may not further distribute the material or use it for any profit-making activity or commercial gain

- You may freely distribute the URL identifying the publication in the public portal 


\section{RESEARCH ARTICLE}

\section{Trading wind power through physically settled options and short-term electricity markets}

\section{Athanasios Papakonstantinou*1 | Georgia Champeri | Stefanos Delikaraoglou ${ }^{2}$ | Pierre Pinson $^{3}$}

\footnotetext{
${ }^{1}$ Department of Management Engineering, Technical University of Denmark (DTU),2800 Kgs. Lyngby, Denmark

${ }^{2}$ EEH-Power Systems Laboratory, Swiss Federal Institute of Technology (ETH),Zurich 8092, Switzerland

${ }^{3}$ Department of Electrical Engineering, Technical University of Denmark (DTU),2800 Kgs. Lyngby, Denmark

Correspondence

*Email: athpapa@dtu.dk
}

\begin{abstract}
Wind power producers participating in today's electricity markets face significant variability in revenue streams, with potential high losses mostly due to wind's limited predictability and the intermittent nature of the generated electricity. In order to further expand wind power generation despite such challenges it is important to maximise its market value, and move decisively towards economically sustainable and financially viable asset management. In this paper, we introduce a decision-making framework based on stochastic optimisation, that allows wind power producers to hedge their position in the market by trading physically settled options in futures markets in conjunction with their participation in the short-term electricity markets. The proposed framework relies on a series of two-stage stochastic optimisation models that identify a combined trading strategy for wind power producers actively participating in both financial and day-ahead electricity markets. The proposed models take into consideration penalties from potential deviations between day-ahead market offers and real-time operation and incorporates different preferences of risk aversion, enabling a trade-off between the expected profit and its variability. Empirical analysis based on data from the Nordic region illustrates high efficiency of the stochastic model and reveals increased revenues for both risk neutral and risk averse wind producers opting for combined strategies.

KEYWORDS:

Trading strategies, electricity markets, futures markets, options, uncertainty, stochastic programming, risk, wind energy.
\end{abstract}

\section{1 | INTRODUCTION}

During the past decade wind energy has expanded significantly its share in power systems, to the degree where it can meet consumption for several consecutive days. However, electricity markets were designed with a focus on fuel based conventional generation with minimum exposure in renewable energy sources (RES). This, in turn makes markets and those that participate in them, vulnerable to the limited predictability and increased variability associated with wind energy 1 . Such exposure can often bring price volatility and increase the cost of regulation due to imbalances between market schedule and realised production; both significant challenges for wind energy's economic sustainability and further expansion.

It is therefore imperative to consider how such challenges can be addressed so that wind energy's full potential is achieved. In the context of the liberalised electricity sector, participating in the futures markets is a viable option that can mitigate exposure to financial risks linked with uncertainty both in generation and consumption. Like with conventional commodities, energy can also be traded in futures markets at a fixed price determined before the settlement of short-term electricity trading floors, i.e. day-ahead, intraday or balancing markets. Specifically, Deng 
and Oren ${ }^{2}$ discuss how several types of financial instruments (e.g. forward contracts, futures and swaps) can be used by generators, load serving entities (utilities) and system operators to mitigate their exposure in market price risks. In the same direction, further integration of options in contracts for demand-side management is proposed in 3 .

Towards a more formal approach that relates to fundamental methods from finance literature, Oren 3 applies the Black-Scholes model by replacing stock prices with the forward market clearing under the assumption of zero dividend (i.e. a zero-risk interest return on the stock). The Black-Sholes mode ${ }^{1}$ is an analytic tool that can determine theoretical prices of specific derivatives over time given the stock price, the associated dividend and a stock volatility measure, but despite its dominance in finance literature, there is not a consensus on how it can be applied on electricity markets. Lane et al. ${ }^{5}$ identify a limitation of the Black-Scholes model as it assumes a log-normal distribution of electricity prices. Instead, they propose an agent-based approach using genetic algorithms to simulate the market participants' valuations as they determine whether to buy or sell an option. Similarly, Bhanot ${ }^{6}$ simulates option prices based on estimates of electricity market prices to demonstrate the impact of consumption uncertainty on electricity markets, using models of Brownian motion to estimate market prices.

Although the aforementioned approaches clearly show that derivatives can be used to contain exposure in price volatility and hedge against uncertainty in electricity prices, they do not identify the steps that a market participant can follow to achieve the discussed positive results. Liu and $\mathrm{Wu}{ }^{7}$ move towards this direction by introducing a risk management decision framework for conventional generators participating in multiple trading floors (i.e. day-ahead and real-time) of an electricity market. Pineda and Conejo ${ }^{89}$ further contribute in this line of work by looking at how conventional generators determine an optimal portfolio of forward contracts and options while considering specific operational constraints such as the availability of production units. They propose a stochastic framework which takes into account three sources of uncertainty: derivatives and day-ahead market clearing prices, and the probability of failure for the production units and demonstrate that options can be more appropriate hedging instruments than forward contracts. Despite the significance of such contributions, they overlook stochastic generation by restricting their analysis to conventional generators. Designing a trading framework to assist wind power producers in their decision making poses a challenging problem as it introduces new sources of uncertainty and additional market layers i.e. real-time balancing markets.

Against this background, the contribution of this paper lies in formulating a trading framework based on stochastic optimisation that determines the optimal trading strategy for wind power producers with varying risk sensitivity, trading options under the requirement that if an option is settled the contracted energy will be generated, in addition to participating in the day-ahead electricity market. This paper extends the state of the art by accounting for more dimensions of uncertainty and considering a realistic representation of an electricity market that consists of multiple trading floors i.e. futures markets, day-ahead scheduling and real-time balancing. Modelling this complex decision-making process would naturally require a multi-stage optimisation framework that could be practically intractable. To address this challenge we decompose a typically multi-stage stochastic optimisation problem to two two-stage stochastic problems. The proposed formulation provides a computationally tractable model, while it establishes an implicit link between trading electricity through derivatives in futures markets and day-ahead scheduling and physical delivery of electricity. An empirical analysis of the proposed framework for a realistic test case utilising historical data of options, electricity market prices and wind power generation for the Nordic region, demonstrates the efficiency of the stochastic model and illustrates the advantage of combined trading in futures and short-term electricity markets, as opposed to only participating in the day-ahead market. The numerical evaluation concludes with an analysis of a risk averse producer's exposure to different levels of risk which reinforces trading options as a risk diminishing strategy that limits exposure to regulation costs by favouring futures to day-ahead markets.

The rest of the paper is organised as follows. In Section 2 we introduce the basic concepts from futures and electricity markets and outline the decision-making process of a wind power producer actively participating in these markets. In Section 3 we identify the sources of uncertainty involved in our problem and outline the process to formulate statistical scenarios compatible with the stochastic optimisation problems also introduced in this section. These stochastic optimisation problems are numerically evaluated in Section 4 showing how the proposed framework can be used for wind power producers operating in the Nordic region. Finally, we conclude in Section 5

\section{2 | THE MARKET FRAMEWORK}

This section introduces the decision-making process for a wind power producer trading options in addition to its participation in the short-term electricity markets. Prior to that, we relay the basic principles behind the financial products and describe a typical market setup that consists of a day-ahead and a real-time trading floor.

${ }^{1} \mathrm{An}$ analysis of the Black-Scholes model exceeds the scope of this paper; as such, further information can be found in finance related literature cf. ${ }^{4}$. 


\section{1 | Derivatives based on electricity}

Derivatives are financial contracts that draw their value from the value of an underlying asset such as a commodity or even another financial instrument. Having electricity as the underlying asset can lead to derivatives that provide hedging opportunities and can reduce the risk of exposure to balancing costs often associated with renewable energy. There are several types of derivatives that offer various advantages and can mitigate different types of risk depending on the terms of the contract.

In this context, a forward contract is an agreement to buy or sell a specific amount of electricity at a certain price at an arranged date in the future known as expiration or maturity date. Any transaction involves two parties, with the party selling the electricity assuming a short position and the party buying it assuming a long position. A producer may accept a contract at a price that is higher than the one expected at the electricity market several days, weeks, months or even years ahead and profit by exercising that contract instead of participating in the actual market. Still, under the terms of the agreement, it is mandatory for the short party to sell the agreed quantity, even if it is not possible to produce it. This can expose producers to additional risks, which however that can be addressed by introducing more complex financial instruments such as options. As opposed to forward contracts, options are non-mandatory agreements that allow their holders to either buy (i.e. call options) or sell (i.e. put options) a specific amount of energy at the expiration date for a fixed price known as exercise or strike price. Both call and put options have long and short sides depending on whether they are bought or sold.

\subsection{The electricity markets}

Electricity markets are more complex than the commodity markets that finance literature refers as spot markets. The real-time component and the very nature of electricity differentiate commodity markets from electricity markets which typically consist of trading floors sequentially cleared at different time horizons. The largest volumes of electricity are traded today in day-ahead markets, cleared 12 to 36 hours before the physical delivery of electricity with producers and consumers submitting offers in the form of price-quantity bids. Once the market is cleared, the operator announces the day-ahead price and an initial generation schedule. Wind power producers comply to the same rules as the conventional ones, only in their case, actual production may deviate from day-ahead schedule given that their offers are based on forecasts.

To cope effectively with the uncertainty in a market driven power system, producers have to consider complex trading strategies that span through the various trading floors in order to mitigate their exposure to increased balancing costs and financial losses due to the deployment of typically expensive reserves. The simplified overview that follows, is based on the components used for this specific work; a detailed analysis of electricity markets and the role of various actors from market operators to transmission and distribution operators can be found in 10 and ${ }^{11}$.

\section{3 | Decision making for wind power producers}

Against this background, we outline the decision-making process of a wind power producer actively participating in both futures and short-term electricity markets. We focus on a simple market setup which consists of a day-ahead market followed by a real-time market to settle energy deviations with respect to the day-ahead schedule.

In this basic framework, a wind power producer aims to maximise its revenue by determining its optimal involvement in both the futures and the day-ahead electricity markets. At the beginning of the planning period, the producer decides on the purchase of a set of financial contracts, with the decision on whether to exercise them or not left closer to delivery, at the day-ahead market. Noting that the day-ahead decisions can be executed at a rolling horizon on a daily basis with hourly resolution before the day-ahead market clears, the decision process consists of a planning period and an active trading period with the following characteristics

- At the planning period ranging from years, months, weeks or days prior to the day of delivery of each contract, the producer decides on the purchase of derivatives which may include a variety of financial instruments at different maturity dates covering an extended period.

- At each hour of the active trading period, which typically concludes the day-ahead market clearing, the producer decides whether or not to exercise the derivative if that is applicable (i.e. option) based on estimates of its actual production and market prices and determines its offer in the day-ahead electricity market. In case of futures at their expiration date, they are exercised based on the terms of the contract.

This process is described in Figure 1 which provides the timeline for participating in all three trading floors. In this context, let a producer purchase a put option on selling $100 \mathrm{MW}$ at $40 € / \mathrm{MWh}$ at the price of $1 € / \mathrm{MWh}$. At the time of maturity the producer chooses whether to exercise it or not depending on its estimate of the day-ahead market clearing. If the market price is expected at $30 € / \mathrm{MWh}$, then the producer will exercise it to receive a payment of $4000 €$ while participating only in the electricity market would have led to a payment of $3000 €$. For a dayahead market price estimated at $50 € / M W h$ the producer expects to yield higher profit if it does not exercise the option. The producer chooses 
to sell its production in the market instead and disregards the $100 €$ paid to acquire the option; an alternative that would not have been available had the producer signed a forward contract instead. In that case, the opportunity to benefit from the increased price would have been missed.

The example intuitively demonstrates the benefits of trading options and the corresponding risks. Increased profits are a clear benefit but they come at a significant risk as it is highly unlikely that the option or market prices will be known prior to the decision-making process. Irrespective of the derivative a wind power producer selects, and despite the wide range of available financial instruments $2^{2}$ there will always be financial risks when trading electricity, as long as there is uncertainty in generating and consuming it. Price volatility follows and even if literature shows that futures markets can reduce exposures to such risks, it acknowledges that additional ones are brought as new sources of uncertainty are introduced.

To address the challenges faced by wind power producers participating in both futures and short-term electricity markets requires a decisionmaking process that accounts for multiple sources of uncertainty. In this context, the proposed framework utilises an approach based on stochastic optimisation to incorporate uncertainty that relates both to wind energy's stochastic nature and to the limited information on market outcomes.

\section{3 | OFFERING STRATEGY FOR A WIND POWER PRODUCER}

In this section we describe how the two-step decision process expands to a multi-stage optimisation problem with each stage corresponding to different points in time when uncertainty is unveiled and different trading floors. We present the intuition behind the reduction of a typically multistage problem to a series of two-stage stochastic optimisation problems and then introduce the mathematical formulation of the trading framework for both risk neutral and risk averse producers.

We consider a wind power producer trading in all the three markets introduced in Section 2 The producer's objective is to maximise profits from purchasing options in the futures markets while actively trading in the day-ahead market based on forecasts of its production and consequently susceptible to losses in the real-time market due to potential imbalances.

\subsection{An optimisation framework for trading wind energy under uncertainty}

The structure of this problem resembles the decision-making process of energy investment and capacity expansion problems, common in the technical literature (for an in-depth introduction to this topic cf. ${ }^{12}$ ). Specifically, the options purchase phase in the wind power trading problem is analogous to the investment decision of a capacity expansion problem, while participation in the short-term electricity market is modelled similarly in both problems. Furthermore, both problems involve high level of uncertainty, e.g. in future electricity prices, that requires proper risk management. In addition, in both cases the decisions to be made are dynamic in a sense that they are made throughout a long planning horizon as more uncertain parameters become known, e.g. the options strike price or the fuel cost of new generation assets.

Given that the problem at hand involves three trading floors spanning a horizon up to a quarter, a reasonable approach would be to formulate a multi-stage stochastic optimisation problem akin to investment decision problems ${ }^{1314}$. However, there are two notable differences between these problems in terms of uncertainty modelling and timing of decisions that prevent the application of a stochastic multi-stage optimisation formulation in practice. Regarding the former aspect, the variability and uncertainty of wind energy in investment problems are typically approximated throughout the planning horizon by duration curves derived from historical data, which are divided into time segments of different production levels. While this approach captures adequately the characteristics of wind energy in a very long-term horizon, e.g. 20 years as the typical lifetime of wind farms, it poorly describes uncertainty in the shorter time horizons that are more common in trading applications. In such cases, profitability is largely driven by real-time forecast errors and the associated imbalance penalties and not long-term investment returns.

Considering the timing of decisions, typical investment problems adopt a static approach, whereby the optimal expansion decisions are obtained for a single representative year, assuming that the rest of the power system characteristics remain unchanged in the future. Unlike power system configuration that may remain relatively unchanged, option prices are much more volatile and thus it is highly essential for the proposed trading strategy to capture this dynamic behaviour. On the other hand, an attempt to model all the uncertainty sources that are involved in our options purchase and energy trading problems in an accurate probabilistic framework preserving also the timing of decision-making would yield a computationally intractable multi-stage stochastic optimisation problem.

To overcome these complexities we propose an alternative approach exploiting some of the properties that stem from the practical implications of the specific problem that is to be addressed. First, we consider that options purchased during a period q to be exercised at a future period $q^{\prime}$ can be exercised at any trading day during $q^{\prime}$. Furthermore, the options' transaction fee is set equal to $0.0045 € / \mathrm{MWh} \mathrm{H}^{3}$ with the price the producer pays

\footnotetext{
${ }^{2}$ Further definitions and examples of several types of derivatives incl. forward contracts and options are found in ${ }^{\sqrt[4]{ }}$. Moreover, Deng and Oren ${ }^{2}$ provide a detailed overview of applications in electricity markets.

${ }^{3}$ This is the trading fee for Nasdaq operated Nordic and European markets; http://www.nasdaqomx.com/transactions/markets/commodities/Marketaccess/feelist accessed at 07.06.18.
} 
to acquire an option set equal to $3 € / \mathrm{MWh}$, a value significantly higher than examples from literature cf. $\frac{8}{\text { in }}$ order to have a complete modelling framework. The option fee and price, denoted by $\lambda^{\mathrm{O}}$ and $\lambda^{\mathrm{p}}$ respectively, are constants that do not impact the decision making process, although they impact the producers' total profits as seen in equation $3 \mathrm{c}$

The aforementioned practical attributes of the employed financial instruments allow us to decouple the multi-stage optimisation problem and reduce it to a series of two two-stage stochastic optimisation programs linking the trading of options with participating in the day-ahead and realtime markets; a process schematically shown in Figure 2 Each of these two stochastic programs pertain to different decisions of the wind power producer, i.e. options purchase and short-term trading subject to imbalance penalties.

In more detail, for the options purchase problem the first-stage decision is the amount of options to be bought today (here-and-now decision) based on the revenues brought by the options exercised at an already known strike price. In turn, the second stage models the possibility to buy options in the remaining of the quarter (wait-and-see decisions), based on the statistical estimation of the strike prices and the expected revenue if they are executed in the active trading period. The amount of options bought during the planning period is not a hard constraint for our problem given that we do not impose any budget constraints in the model as long as we compose an options' portfolio with favourable strike prices that can be exercised in the next period. This optimisation model is implemented as a receding horizon scheme with daily time-step, i.e. at each day only the first stage of the purchase sequence is executed, while the rest are discarded.

The short-term trading stochastic optimisation problem that follows, is implemented on a daily basis as it models a wind power producer's participation in the short-term markets. In the beginning of each trading day and for 24 different instances, assuming hourly trading periods, the model determines the producer's optimal trading position each period. Here, we consider as first-stage decisions the day-ahead market offer and the amount of options to be exercised, and as second-stage decisions the amount of energy that has to be sold/purchased in the balancing market to cover deviations from the day-ahead schedule due to forecast errors. In this model, second-stage decisions are subject to uncertainty in terms of balancing prices and the actual realisation of wind power production, due to the 12-36 hours time window between the gate closure of the day-ahead market and the actual delivery of electricity.

The efficiency of the proposed trading framework and consequently its usefulness to a wind power producer heavily depends on the accuracy of models used to describe the underlying stochastic processes. There are three sources of uncertainty: i) option strike prices, ii) wind power realisation and iii) imbalance costs based on estimates of day-ahead and regulation prices, with i) affecting the option purchase problem and ii) and iii) the short-term trading one. For both stochastic optimisation models we generate two distinct scenario sets that represent different realisations of the random variables that correspond to the sources of uncertainty in our model using methods that are presented in detail in Section 4.2

In this context, as both stochastic optimisation problems are brought together to form the decision-making framework, it becomes apparent that the short-term trading problem takes into consideration the information that becomes available in the periods after the options have been purchased by using the output of the option purchase problem as its input. This, allows us to model the whole decision-making process as a series of two-stage stochastic programs instead of a single multi-stage stochastic model, and therefore we can sidestep the practical caveat of modelling wind power and market price uncertainties for an unnecessary long time horizon as the options purchase problem requires and only considers these uncertainties when they really become relevant and can be actually modelled efficiently. This formulation is in line with the existing literature cf. $\frac{15}{15}$ as for both optimisation problems each stage represents a step from the two-step decision process with the here-and-now decisions made during a planning period and the wait-and-see decisions made after the stochastic processes are realised.

\section{2 | Mathematical formulation}

Despite modelling the decision process as two distinct but interconnected optimisation problems, there are some assumptions that apply to the whole model, in addition to the assumptions for each stage. Specifically, we assume that producers behave as price-takers in markets exhibiting perfect competition. That is, options' prices, and day-ahead and real-time market clearings are not affected by the producers' decisions. This fundamental assumption is common in the relevant literature cf.161011 where perfect competition is assumed for electricity markets with the producers not capable of influencing the market outcome.

In the option purchase problem, we omit more speculative financial instruments by only considering long positions (i.e. purchases) on physically settled put options. Given that our analysis centres on wind power producers trading their actual production in electricity markets, we focus on the financial instruments that will give them additional choices by allowing them to sell their estimated production either on futures or day-ahead market.

Finally, regarding real-time markets, we assume that wind power producers are not capable of offering ancillary services. That is, they do not actively bid in regulation or reserve markets and do not apply control strategies on their real-time production.

\section{The option purchase problem}

In the beginning of each day $d_{1}$, a wind power producer has to decide on the number of options that should be purchased for the next period. 
Although the producer has access to an option's strike price $\lambda_{\mathrm{d}_{1}}^{\mathrm{S}}$ for the present day, it still faces uncertainty regarding the evolution of option prices $\lambda_{d}^{S}$ in the remaining days of the planning period $d=\{2, \ldots, D\}$. To account for this source of uncertainty, future options' strike prices are considered as random variables and modelled using a set of scenarios $\Omega$ such that $\lambda_{\mathrm{d} \omega}^{\mathrm{S}}$ is the strike price at day d and scenario $\omega$ with corresponding probability of occurrence $\pi_{\omega}$. Then, the optimal number of options $x_{d_{1}}$ to be bought in day $d_{1}$ as well as the amount of options $x_{d} \omega$ that can be purchased over the following days depending on price uncertainty outcome $\omega$, are given as the solution to the following two-stage optimisation problem

$$
\max _{x_{d_{1}}, x_{d} \omega} \lambda_{d_{1}}^{S} x_{d_{1}} E_{o}+\sum_{d=2}^{D} \sum_{\omega=1}^{\Omega} \pi_{\omega} \lambda_{d \omega}^{S} x_{d \omega} E_{o}
$$

subject to

$$
\begin{aligned}
& 0 \leq \mathrm{x}_{\mathrm{d}_{1}} \leq \mathrm{X}, \\
& 0 \leq \mathrm{x}_{\mathrm{d} \omega} \leq \mathrm{X}, \forall \mathrm{d}, \forall \omega, \\
& \mathrm{x}_{\mathrm{d}_{1}}+\sum_{\mathrm{d}=2}^{\mathrm{D}} \mathrm{x}_{\mathrm{d} \omega} \leq \mathrm{X}^{\mathrm{tot}}, \forall \omega .
\end{aligned}
$$

The objective function $1 \mathrm{a}$ t to be maximised is the expected revenue from possibly exercising the purchase options, where $E_{o}$ denotes the size (in terms of energy volume) of option $o \in \mathrm{O}$. The expected revenue relates to the options' purchase schedule, with the short-term term trading models that follow determining whether they will be actually exercised or not. Constraints $1 \mathrm{~b}$ ] and $1 \mathrm{c}$ ] set an upper limit $X$ on the amount of options purchased each day. Constraint $1 \mathrm{~d}$ ] states that the total number of options purchased for the whole planning horizon and for every scenario $\omega$ must be at most equal to $\mathrm{X}^{\text {tot }}$. In practice, this is a predefined parameter specified by the wind power producer according to the size and the capacity factor of its portfolio as well as the options' size $E_{o}$.

It is important to clarify that model [1] is executed in a daily rolling horizon until the end of the planning period or until the maximum number of purchased options $X^{\text {tot }}$ is reached. This allows to refine strike price information over time and incorporate it in decision-making using an updated set of price scenarios. Given the lengthy decision horizon of the overall purchase process, i.e. up to a quarter in the current implementation, it is plausible that long-term strike price dynamics are not accurately captured in the scenario set. This flaw, which stems from the nature of problem, can be addressed by the proper selection of the daily purchase limit $X$ to exploit favourable short-term strike prices but leaving a buffer on the remaining number of options to be purchased at better prices which are not captured by the current scenario set. According to this procedure, the value of parameter $X^{\text {tot }}$ has to be updated on a daily basis subtracting the number of options bought the previous day.

\section{Short-term trading strategy of a risk neutral wind power producer}

Given the portfolio of options bought during the planning period based on model 11, the wind power producer has to decide how to trade its energy production for the next day. In particular, the producer has to determine the amount of energy to be sold either in the day-ahead market or through options, taking into account the penalties from the balancing market due to wind power production uncertainty. Aiming at maximising its expected profit from participating in these trading floors, a wind producer solves the following two-stage stochastic program

$$
\max _{\mathrm{p}_{\mathrm{t}}^{\mathrm{O}}, \mathrm{p}_{\mathrm{t}}^{\mathrm{D}}, \Delta \mathrm{p}_{\mathrm{t} \omega}^{+}, \Delta \mathrm{p}_{\mathrm{t} \omega}^{-}} \sum_{\mathrm{t}=1}^{\mathrm{T}}\left[\lambda_{\mathrm{t}}^{\mathrm{S}} \mathrm{p}_{\mathrm{t}}^{\mathrm{O}}+\hat{\lambda}_{\mathrm{t}}^{\mathrm{D}} \mathrm{p}_{\mathrm{t}}^{\mathrm{D}}+\sum_{\omega=1}^{\Omega} \pi_{\omega}\left(\lambda_{\mathrm{t} \omega}^{\mathrm{D}} \mathrm{r}_{\mathrm{t} \omega}^{+} \Delta \mathrm{p}_{\mathrm{t} \omega}^{+}-\lambda_{\mathrm{t} \omega}^{\mathrm{D}} \mathrm{r}_{\mathrm{t} \omega}^{-} \Delta \mathrm{p}_{\mathrm{t} \omega}^{-}\right)\right]
$$

subject to

$$
\begin{aligned}
& \mathrm{p}_{\mathrm{t}}^{\mathrm{D}}+\mathrm{p}_{\mathrm{t}}^{\mathrm{O}} \leq \mathrm{W}, \forall \mathrm{t}, \\
& \mathrm{p}_{\mathrm{t}}^{\mathrm{O}}=\sum_{\mathrm{o}=1}^{\mathrm{O}} \mathrm{u}_{\mathrm{to}} \mathrm{n}_{\mathrm{to}} \mathrm{E}_{\mathrm{o}}, \forall \mathrm{t}, \\
& \mathrm{n}_{\mathrm{to}} \leq \mathrm{N}_{\mathrm{o}}, \forall \mathrm{t}, \forall \mathrm{o}, \\
& \Delta \mathrm{p}_{\mathrm{t} \omega}^{+}+\Delta \mathrm{p}_{\mathrm{t} \omega}^{-}=\mathrm{P}_{\mathrm{t} \omega}-\left(\mathrm{p}_{\mathrm{t}}^{\mathrm{D}}+\mathrm{p}_{\mathrm{t}}^{\mathrm{O}}\right), \forall \mathrm{t}, \forall \omega, \\
& \Delta \mathrm{p}_{\mathrm{t} \omega}^{+} \leq \mathrm{P}_{\mathrm{t} \omega}, \forall \mathrm{t}, \forall \omega, \\
& \Delta \mathrm{p}_{\mathrm{t} \omega}^{-} \leq \mathrm{W}, \forall \mathrm{t}, \forall \omega, \\
& \mathrm{p}_{\mathrm{t}}^{\mathrm{O}}, \mathrm{p}_{\mathrm{t}}^{\mathrm{D}} \geq 0, \mathrm{n}_{\mathrm{to}}, \forall \mathrm{t}, \forall \mathrm{o}, \\
& \Delta \mathrm{p}_{\mathrm{t} \omega}^{+}, \Delta \mathrm{p}_{\mathrm{t} \omega}^{-} \geq 0, \forall \mathrm{t}, \forall \omega, \\
& \mathrm{u}_{\mathrm{to}} \in\{0,1\}, \forall \mathrm{o} .
\end{aligned}
$$


The objective function 2a to be maximised is the total expected profit of the wind power producer. The first stage decision $p_{t}^{O}$ is the amount of energy to be sold exercising available options with strike price $\lambda^{\mathrm{S}}$. Similarly, the decision variable $\mathrm{p}_{\mathrm{t}}^{\mathrm{D}}$ is the energy volume traded in the dayahead market at the expected spot price $\hat{\lambda}_{t}^{D}$. Note that regarding first-stage decisions, spot price uncertainty can be summarised in a single-valued forecast, i.e. expected value, under the assumption that wind power production and day-ahead market price are not correlated; an assumption in line with a setting of perfect competition in which wind producers cannot influence market prices. The second-stage variables $\Delta \mathrm{p}_{\mathrm{t} \omega}^{+}$and $\Delta \mathrm{p}_{\mathrm{t} \omega}^{-}$ represent the excess and deficit of energy production with respect to the scheduled quantities, respectively. Parameters $r_{t \omega}^{+}$and $r_{t \omega}^{-}$are the ratio of the positive and negative imbalance prices respectively with the day-ahead market price $\lambda_{\mathrm{t} \omega}^{\mathrm{D}}$ in period $\mathrm{t}$ and scenario $\omega$.

Constraint $2 b$ ensures that the level of contracted energy through options and the day-ahead market is lower or equal to the installed capacity $\mathrm{W}$ of the wind energy portfolio. This assumption highlights the responsibility of the decision maker as a participant in both futures and short-term electricity market with a link in actual production and not just a speculative force acting only on financial markets. In this context, the energy sold through options is defined according to constraint $2 \mathrm{C}$, where $n_{t o}$ and $E_{o}$ denote the number and size of options units and the binary variable $\mathrm{u}_{\text {to }}$ is equal to 1 if option o is exercised in period t or 0 otherwise. Constraint 2d] limits the number of options exercised at each time period to the number $\mathrm{N}_{\mathrm{o}}$ of the corresponding options purchased. The positive $\Delta \mathrm{p}_{\mathrm{t} \omega}^{+}$or negative $\Delta \mathrm{p}_{\mathrm{t} \omega}^{-}$deviations from the contracted amount of energy are calculated according to equation $\left[2 \mathrm{e}\right.$, based on the realised wind power production $\mathrm{P}_{\mathrm{t} \omega}$ for each scenario $\omega$ and period $\mathrm{t}$. The split of energy imbalance to positive and negative deviations requires that the imbalance penalties are $r_{t \omega}^{+} \geq 1$ and $r_{t \omega}^{+} \leq 1$. This property, which follows from the Nordic balancing market structure, ensures that at the optimal solution either $\Delta \mathrm{p}_{\mathrm{t} \omega}^{+}$or $\Delta \mathrm{p}_{\mathrm{t} \omega}^{-}$will be equal to zero. Constraints 2f] and 2g. set the upper bounds of the positive and negative imbalance equal to the wind power realisation and the installed capacity, respectively. Finally, constraints 2h-2j] are variable declarations.

\section{Short-term trading strategy of a risk averse wind power producer}

Trading model 2] presented above aims at maximising the expected profit of a risk neutral wind power producer. This formulation does not account though for the variance of revenue as well as about its dispersion from the expected value. However, it is often the case where a wind power producer is not only interested in the expected value of its profit but also in the probability of experiencing heavy losses due to possibly high imbalance penalties 11718 .

A common risk measure to account for the risk aversion in trading strategies is the conditional value at risk (CVaR). The CVaR at confidence level $\alpha \in[0,1)$ is defined as the mean value of the $1-\alpha$ cases with lowest profit. The main advantage of CVaR compared to other risk measures proposed in the technical literature, is its mathematical coherence $\frac{19}{19}$ and its ability to take into account fat tails, i.e. probability distributions with large skewness or kurtosis. CVaR is incorporated in the trading strategy model as follows

$$
\max _{\mathrm{p}_{\mathrm{t}}^{\mathrm{O}}, \mathrm{p}_{\mathrm{t}}^{\mathrm{D}}, \Delta \mathrm{p}_{\mathrm{t} \omega}^{+}, \Delta \mathrm{p}_{\mathrm{t} \omega}^{-}} \Pi_{\omega}+\beta\left(\zeta-\frac{1}{1-\alpha} \sum_{\omega=1}^{\Omega} \pi_{\omega} \eta_{\omega}\right)
$$

subject to

$$
\begin{aligned}
& \text { constrains } 2 \mathrm{~b}-2 \mathrm{j} \text {, } \\
& \Pi_{\omega}=\sum_{\mathrm{t}=1}^{\mathrm{T}}\left[\lambda_{\mathrm{t}}^{\mathrm{S}} \mathrm{p}_{\mathrm{t}}^{\mathrm{O}}+\hat{\lambda}_{\mathrm{t}}^{\mathrm{D}} \mathrm{p}_{\mathrm{t}}^{\mathrm{D}}+\sum_{\omega=1}^{\Omega} \pi_{\omega}\left(\lambda_{\mathrm{t} \omega}^{\mathrm{D}} \mathrm{r}_{\mathrm{t} \omega}^{+} \Delta \mathrm{p}_{\mathrm{t} \omega}^{+}-\lambda_{\mathrm{t} \omega}^{\mathrm{D}} \mathrm{r}_{\mathrm{t} \omega}^{-} \Delta \mathrm{p}_{\mathrm{t} \omega}^{-}\right)\right] \text {, } \\
& -\Pi_{\omega}+\zeta-\eta_{\omega} \leq 0, \forall \omega, \\
& \eta_{\omega} \geq 0, \forall \omega \text {. }
\end{aligned}
$$

The new objective function $3 a$ is a trade-off between the expected profit and the CVaR of the profit distribution. The decision maker can adjust this trade-off by selecting the value of the weighting parameter $\beta$ according to its risk aversion level. In fact, for $\beta=0$ the objective function $3 a$, becomes identical to one of the risk neutral formulation in $2 \mathrm{a}$. On the contrary, higher values of $\beta$ indicate more risk averse behaviour, since the CVaR term becomes more important. Choosing different values of $\beta$ allows to draw the efficient frontier, i.e. a set of points (expected profit, risk) that none of them is strictly dominant to the others. Constraint $3 \mathrm{c}$ defines the profit for each scenario $\omega$ and constraints $3 \mathrm{~d}$ ] and $3 \mathrm{e}$ along with the auxiliary variables $\zeta$ and $\eta_{\omega}$ are used to model the CVaR.

\section{Short-term trading strategy as a deterministic problem}

For the formulation of the day-ahead market participation as a deterministic problem we summarise wind power's uncertainty into a single-valued forecast, such as the stochastic process's conditional expectation $\hat{W}_{\mathrm{t}}$. While such trivialisation of the forecasting process cannot be considered as an original contribution, it does act as a benchmark for the proposed trading framework given that the deterministic model does not account for potential imbalance penalties due to forecast errors. 
In this context, participation in the day-ahead market is formulated as the following optimisation problem

$$
\max _{\substack{\mathrm{O} \\ \mathrm{p}_{\mathrm{t}}, \mathrm{p}_{\mathrm{t}}^{\mathrm{D}}}} \sum_{t=1}^{\mathrm{T}}\left[\lambda^{\mathrm{S}} \mathrm{p}_{\mathrm{t}}^{\mathrm{O}}+\hat{\lambda}_{\mathrm{t}}^{\mathrm{D}} \mathrm{p}_{\mathrm{t}}^{\mathrm{D}}\right]
$$

subject to

$$
\begin{aligned}
& \mathrm{p}_{\mathrm{t}}^{\mathrm{D}}+\mathrm{p}_{\mathrm{t}}^{\mathrm{O}}=\hat{\mathrm{W}}_{\mathrm{t}}, \forall \mathrm{t}, \\
& \mathrm{p}_{\mathrm{t}}^{\mathrm{O}}=\sum_{\mathrm{o}=1}^{\mathrm{O}} \mathrm{u}_{\mathrm{to}} \mathrm{n}_{\mathrm{to}} \mathrm{E}_{\mathrm{o}}, \forall \mathrm{t}, \\
& \mathrm{n}_{\mathrm{to}} \leq \mathrm{N}_{\mathrm{o}}, \forall \mathrm{t}, \forall \mathrm{o}, \\
& \mathrm{p}_{\mathrm{t}}^{\mathrm{O}}, \mathrm{p}_{\mathrm{t}}^{\mathrm{D}} \geq 0, \mathrm{n}_{\mathrm{to}}, \forall \mathrm{t}, \forall \mathrm{o}, \\
& \mathrm{u}_{\mathrm{to}} \in\{0,1\}, \forall \mathrm{o} .
\end{aligned}
$$

The objective function 4a to be maximised is the profit of the wind power producer from trading its production in the day-ahead market and through options. Constraint 4b] states that the amount of energy sold in the day-ahead market plus the total energy sold through options each time period $t$ is equal to the expected wind power production $\hat{W}_{t}$. Constraints $4 \mathrm{c}$ - $4 \mathrm{f}$ remain the same as in the stochastic trading strategy 2 .

\section{Total expected profit for trading options and participating in short-term electricity markets}

At the beginning of the active trading period, the maximum number of options $\mathrm{N}_{\circ}$ that can be exercised are equal to the total units purchased during the previous quarter according to model 1]. For every consecutive day this parameter is reduced by the number of units exercised the day before. The stochastic models 2 and 3, and the deterministic one 4, are executed each day of the active trading period in a rolling planning fashion, with look-ahead optimisation horizon of one day, using price and wind power production scenarios as they update daily.

Based on models 2, [3] and [4], the wind power producer decides on how to utilise the options purchased in model [1] by determining whether to sell its energy production through these options or the day-ahead market. The producer's total expected profit $\Pi_{\text {Total }}^{\exp }$ is given by

$$
\Pi_{\text {Total }}^{\text {exp }}=\sum_{d=1}^{D} \Pi_{d}^{\text {exp }}-\left(\lambda^{O}+\lambda^{p}\right) \sum_{o=1}^{O} N_{o} E_{o}
$$

where $\Pi_{d}^{\text {exp }}$ is the daily expected profit from models [2], 3] and 4], $\lambda^{O}$ the option fee, $\lambda^{p}$ the option premium, and $N_{\circ}$ and $E_{\circ}$ the number and size of each of the purchased options o $\in \mathrm{O}$ respectively.

\section{4 | A CASE STUDY FOR THE NORDIC MARKETS}

In this section we evaluate the proposed model based on a case study for the Nordic region. We first outline the Nordic electricity market setup and then discuss how data requirements were met regarding scenario generation given the multiple sources of uncertainty. We then provide a detailed evaluation of the decision-making process for the time period that includes the last three quarters of 2006 and the first three of 2007 and outline the benefits of combined trading in both futures and day-ahead markets. Finally, we provide a risk analysis and demonstrate the impact of risk aversion in the producers' revenue.

\subsection{The Nordic electricity markets}

The Nordic electricity market consists of multiple trading floors depending on the time-horizon at which electricity is traded in connection with physical power delivery. As shown in Figure 3 there are four types of markets that define the Nordic setting.

At a long-term horizon spanning from years to days prior to delivery, electricity is traded through financial instruments which are bought and sold in futures markets. In such markets participants trade financial products linked to a specific commodity. Electricity is treated as such a commodity bereft of the characteristics that are associated with the power system (i.e. power balance, voltage, frequency control). Electricity derivatives for the Nordic region, are traded in Nasdaq Nordic (formerly known as Nasdaq OMX).

The link with the power system is established closer to delivery for those trading in the day-ahead market. For the Nordic region such market is operated by Nord Pool. In Nord Pool's day-ahead market (Elspot) electricity suppliers and consumers with physical access to the power grid place their offers which are aggregated to supply and demand curves. The market clearing price for each of the following day's 24 hours is the intersection of the two curves. 
The significant time interval of 12 to 36 hours between Elspot participation and physical operation for the renewable heavy Nordic countries can potentially lead to significant deviations between day-ahead scheduling and physical delivery of electricity. In order to alleviate such deviations, Nord Pool also operates an intraday market (Elbas), a continuous matching market operating at first-come, first-serve basis, which nevertheless exhibits limited liquidity 20 .

Once Elspot and Elbas markets are processed, there can be still power imbalances between electricity generation and consumption as we move towards the delivery time. Responsible for mitigating such deviations and restoring system balance is the Transmission System Operator (TSO) through the real-time market.

\subsection{Data requirement and scenario generation}

The proposed trading framework links trading futures with the day-ahead and real-time markets. As expected, the scenarios for both option purchase and short-term trading problems require data from all three markets.

\section{Derivatives}

We consider six quarterly option contracts from Q2 2006 to Q3 2007. The volume of each option is set equal to 2190 MWh for the quarterly interval which in turn consists of around 2190 hours given the number of days in each month including winter time or summer time and leap years, and a base contract size of $1 \mathrm{MW}^{21}$.

The scenarios for the expected daily option strike prices are generated using an auto-regressive integrated moving average (ARIMA) model to describe options' price dynamics and uncertainty over time. The ARIMA model characterises the uncertainty regarding the strike price of the derivative of interest (i.e. a quarterly put option on price area DK1) based on historical data. This approach is widely adopted in energy price forecasting applications and formally defined in the context of scenarios for stochastic optimisation in ${ }^{8}$ and ${ }^{11}$ among several sources.

Based on historical data from 02/01/2004 to 31/03/2006 we generate 20 scenarios for the daily strike prices for each decision period. For each of the quarterly intervals, the expected prices are generated based on the data available prior to that day with each new day's scenarios taking into account the realisation of the price the day before.

\section{Day-ahead market}

We consider a set of expected prices to model the uncertainty a wind power producer faces regarding the day-ahead market clearing price, given that it has to make a decision on its position prior to market clearing. Therefore, we generate 20 scenarios for the day-ahead market participation stochastic problem based on historical Elspot prices for the period 01/04/2006 until 30/09/2007 using a simple approach inspired by the literature related to wind energy trading under uncertainty in which price stochasticity is estimated based on historical information without significant loss in forecast accuracy 22, 1 . For the purposes of the proposed setting, each one of the 20 day-ahead market price scenarios can be traced back to a respective week using a sliding window of 20 weeks. For example, scenario $\omega_{1}$ considers Elspot prices one week prior to the decision, scenario $\omega_{2}$ goes two weeks back and so on.

\section{Real-time market}

Regarding real-time operation the model requires forecasts of prices for upward and downward regulation and realisations of wind power production. In principle, the ARIMA model could have been used to generate scenarios for the regulation prices based on historical data which could then be used to calculate the ratio between regulation prices and the day-ahead market price. However, data was not available for the period before the planning and settlement stages as required for training the ARIMA model. Instead, we exploit the seasonality in regulation prices and generated data for 2005 based on histograms of the ratios for 2006, 2007 and 2008. We generate the scenarios by sampling from a Beta distribution that fitted the synthesised data for 2005.

Finally, for the wind power production for the period between 01/01/2006 and 24/10/2007 we use data from the Western Denmark area (DK1) collected by the Danish TSO (Energinet.dk) and processed in 23. Wind power measurements were originally available for approximately 400 geographically distributed grid connection points aggregated into 5 zones with the data set consisting of point forecasts, quantile forecasts and the actual power measurements for each zone and for the whole DK1 area, normalised by the available capacity of each zone at each time. In this paper we consider aggregated zone 5 as a single wind power producer and use the quantile forecasts to generate scenarios based on the methodology outlined in ${ }^{24}$ and 25 . Wind power scenarios are issued based on non-parametric probabilistic forecasts while the interdependence structure of forecast errors among a set of prediction horizons is captured using Copula theory 2624 . These statistical scenarios are derived from probabilistic forecasts of wind power production. The temporal interdependence structure of forecast errors is captured by a covariance matrix estimated using a transformation of prediction errors to Gaussian multivariate random variables. Long-term variations of this covariance matrix are accounted using an exponential forgetting scheme. 
It should be noted that the scenario generation methods we employ represent only parts of the literature, with the empirical evaluation of the proposed framework not depending on the used forecasting techniques. While the contribution of this paper lies in the trading framework itself, we acknowledge that there are several approaches of different complexity that can be used to generate scenarios. For example, 1 generate price scenarios based on historical data to estimate the mean, variance and correlation between prices of different trading floors, a simpler method to the introduced ARIMA process. Still, irrespective of the methods used to generate scenarios, their complexity and the accuracy they may bring in generating realistic outcomes for the decision makers, sometimes it may be impossible to employ them due to their reliance on historical data which may be unavailable for a specific time period. Specifically for this study, the historical period was imposed based on such restrictions on the availability of data for all three markets, namely for the option contracts

In such cases, decision makers have to make assumptions and synthesise data so that the generated scenarios maintain statistical significance. In this context,, 8 generate prices for forward contracts based on the their scenarios for market prices under the assumption that close to power delivery the prices of forward contracts converge to the market prices while taking note that more advanced forecasting methods can generate more accurate scenarios.

Having derived the scenarios for the two stochastic optimisation problems we now proceed to evaluate the model.

\section{3 | Empirical evaluation}

The numerical simulation of the trading framework consists of two parts. For the first part, we assume a risk neutral decision maker and assess the impact of its participation in both futures and day-ahead markets against participating only in the day-ahead market. We evaluate the proposed framework described by optimisation problems [1] and [2] using two cases to denote a lower and an upper bound for the performance of the model. The lower bound is defined by the deterministic optimisation model [4], while for the upper bound we assume that it is possible for the decision maker to have access to perfect information regarding the involved uncertainties, thus eliminate regulation costs. For the second part, we evaluate the option purchase and short-term trading problems assuming a risk averse decision maker as described by optimisation models [1] and [3] to demonstrate the impact of risk control on the decision-making process.

To evaluate the model's efficiency against uncertainty, we introduce a simple two-step process. First, we obtain the decisions made at time step $t$ by solving the main stochastic optimisation models [1] and 2] for all time steps leading up to $t-1$. For the second step, we use the solution already acquired for time step $t$ against the observed outcome from the given data. By iteratively implementing this process for time steps $t>1$ we obtain a set of revenues that includes the estimated outcome of the model and the actual ones based on the observed outcomes. Based on a process described in ${ }^{22}$ we use these revenues to assess the trading strategies through the 'performance ratio' given by

$$
\gamma=\frac{R_{\mathrm{O}}+R_{\mathrm{DA}}+R_{\mathrm{B}}}{R_{\mathrm{DA}+\mathrm{O}}^{*}}
$$

where $R_{O}, R_{D A}, R_{B}$, are the revenues from trading options and participating in day-ahead and real-time markets respectively. $R_{D A+O}^{*}$ is the normalisation factor that denotes the revenue a producer would have obtained had its forecast been equal to the actual realisation. Due to this normalisation, $\gamma$ takes values in $(-\infty, 1]$. Naturally, the ideal outcome would be $\gamma=1$ corresponding to perfect forecasts whereby all market deviations are equal to 0 .

Now, regarding wind capacity, in the Danish context the average wind capacity factor of onshore wind farms located in Denmark during 2006 and 2007 was around $20 \%$ and $25 \%$, respectively. In this context, the nominal capacity of the wind power portfolio considered is $258 \mathrm{MW}$, which corresponds to an average quarterly wind power production equal to $125 \mathrm{GWh}$. Finally, we assume that a producer can purchase up to 60 options each quarter, capped at 5 options daily, setting the model constraints $X^{\text {tot }}$ and $X$ to 60 and 5 respectively. The model's sensitivity to both assumptions is further examined in this section.

Initially, the main benefits of purchasing electricity options in addition to participating in the day-ahead market are outlined in Table 1 which shows a clear advantage of combined trading for all models, irrespective of how (or whether) they model uncertainty. In particular, combined trading yields $20.7 \%$ and $15 \%$ additional revenue for the deterministic and stochastic models respectively and an increase of $13.4 \%$ for decision makers with access to perfect information.

Combined participation in both futures and day-ahead markets and the impact of uncertainty are detailed in Table 2 The amount of energy traded in each market under perfect foresight is less compared to the energy traded in the stochastic model and more compared to the deterministic model, mainly due to the number of exercised options and consequently due to the energy traded through options. Still, a producer maximises its revenue under perfect foresight by avoiding the regulation costs that are bound to occur under uncertainty. Overall, the stochastic model achieves $2 \%$ less revenues compared to perfect information, with the deterministic model resulting in $7 \%$ less revenues. Consequently this translates to performance ratio equal to $97.68 \%$ and $92.48 \%$ for the stochastic and deterministic models, respectively.

For the numerical evaluation of the option purchase problem (cf. model 1]) quarterly purchase limit $\mathrm{X}^{\text {tot }}$ and daily limit $\mathrm{X}$ have been arbitrarily set equal to 60 and 5 respectively. Determining these values reflects on the intuition regarding physical and economic aspects of the option purchase problem and has a clear impact on the outcome of the model. Figure 4 shows sensitivity on the daily purchase limit, with the strike price increasing 
as the daily limit decreases. Furthermore, prices increase as the block of options purchased at the beginning of each quarter increases. However, this clear trend regarding the strike prices is not reflected on the total revenues. Solving again the options purchase and short-term trading problems from models 1 and 2] for various quarterly and daily limits, shows that for all values of quarterly blocks, revenues decrease as the daily limit increases, while revenues increase as quarterly blocks increase (Table 3.

For the second part of the analysis we consider different risk preferences in the decision-making process with a producer now considering the impact of extreme scenarios on its expected profit due to very high imbalance costs. Replacing the short-term trading model [2] with [3] leads to risk aware trading strategies for risk averse decision makers. The trading framework, now consisting of models [1] and [3], is evaluated with $\alpha=0.95$ against the weight parameter $\beta$ that measures risk aversion. As shown in Figure 5 and Table 4 increasing risk aversion decreases the expected total revenues and increases the CVaR. Specifically, the expected revenue of a risk averse producer $(\beta=20)$ is $1.57 \mathrm{~m}$. $€$ or $5.7 \%$ less than that of a risk neutral one $(\beta=0)$, although the risk of profit variability is reduced as indicated by the significantly larger (120\%) value of the CVaR term (Figure 5 . Further analysis of how energy is distributed between options and the day-ahead market (Table 4 reveals that as risk aversion increases, more energy is traded as options and less is traded in the day-ahead market. This is justified by risk averse producers preference in trading options given that it is an action that incurs no losses at that stage. On the contrary, participating in the day-ahead market may lead to losses if the clearing price is lower than the option strike price. In addition, by reducing its participation in the day-ahead, a producer reduces its exposure to regulation costs due to imbalances between day-ahead scheduling and real-time operation.

The model was evaluated using an Intel i7 4-core processor clocking at $3.4 \mathrm{GHz}$ with $8 \mathrm{~GB}$ of RAM running CPLEX 12.6 under GAMS. The computational time for the short-term trading model for a risk neutral producer was 27 minutes. This is the total computational time covering 540 runs of the model as it calculates the producer's profit through 6 quarters.

\section{5 | CONCLUSIONS}

This paper presents a trading framework to support decision making of wind power producers trading financial products and participating in the day-ahead and real-time electricity markets. Based on stochastic programming, the model accounts for three sources of uncertainty, i.e. option prices, wind power production and imbalance costs from the deviation between estimated and actual production, while it spans through three trading floors and their corresponding time horizons. Ideally, these decision-making processes would have been formulated as multi-stage problems, albeit specific complexities related to the dynamic interaction between futures and short-term electricity markets called for a different approach.

The main contribution of this work is the formulation of an optimisation framework that incorporates both financial and short-term electricity markets accounting for various sources of uncertainty involved in each stage. Exploiting the properties of the futures market, we tackle the wind power offering problem in a practical manner by solving a series of two-stage stochastic programs that preserve an implicit link between the different types of markets in a computationally tractable formulation. Initially, the option purchase problem determines the number of options to be purchased each quarter. In turn, the short-term trading problem determines the number of options to be exercised and the day-ahead market offers each day of the following quarter.

In addition to its methodological merit, the proposed formulation leads to a computational tractable model that allows an extensive empirical evaluation of the decision-making framework. In this context, numerical simulations based on data from the Nordic region in the period during 2006-2007, show that a wind power producer, irrespective of its risk sensitivity, can increase its revenues by implementing a trading strategy that combines participation in both futures and day-ahead markets. Moreover, for risk averse producers, it is shown that they increase their revenues by trading heavily in the futures market and limiting their position in the day-ahead market. The scenario generating methods used for the numerical solution of the stochastic optimisation problems represent with adequate accuracy the involved uncertainty. While we acknowledge that more advanced scenario generation methods could have been considered, there is a clear indication on the positive impact of combined participation in both markets.

Nevertheless, for future lines of work the option purchase and short-term trading models can be further improved so that they provide a more accurate and more realistic decision framework. Further future work involves the option purchase problem, which instead of a fixed daily option purchase step, quantitative methods based on historical data could determine whether to purchase all available options based on the daily prices.

\section{ACKNOWLEDGMENT}

Athanasios Papakonstantinou and Pierre Pinson have been partly supported by the Danish Council for Strategic Research (DSF) through the project '5s-Future Electricity Markets', No. 12-132636/DSF. The authors are very grateful to Janne Peljo and Anna Sahari for sharing the data on option prices they gathered for Janne's MSc Thesis 27 . 


\section{References}

1. Botterud A., Zhou Z., Wang J., et al. Wind power trading under uncertainty in LMP markets. IEEE Transactions on Power Systems. 2012;27(2):894-903.

2. Deng S.J., Oren S. S.. Electricity derivatives and risk management.. Energy Economics. 2006;31(6-7):940-953.

3. Oren S. S.. Integrating real and financial options in demand-side electricity contracts. Decision Support Systems. 2001;30:279-288.

4. Hull J. C.. Option, Futures and Other Derivatives. Pearson; 9 ed.2015.

5. Lane D.W., Richter C.W., ShebIÃl' G.B.. Modeling and evaluating electricity options markets with intelligent agents. In: ; 2000.

6. Bhanot K.. Value of an option to purchase electric power âĂŤ the case of uncertain consumption. Energy Economics. 2002;24:121-137.

7. Liu M., Wu F. F.. Risk management in a competitive electricity market. Energy Economics. 2007;29(9):690-697.

8. Pineda S., Conejo A. J.. Managing the financial risks of electricity producers using options. Energy Economics. 2012;34:2216-2227.

9. Pineda S., Conejo A. J.. Using electricity options to hedge agains financial risks of power producers. Journal of Modern Power Systems and Clean Energy. 2013;1:101-109.

10. Conejo A. J., Carrión M., Morales J. M.. Decision Making Under Uncertainty in Electricity Markets. Springer; 2010.

11. Kirschen D., Strbac G.. Fundamentals of Power System Economics. John Wiley \& Sons, Ltd; 2004.

12. Siddiqui A. S., Kazempour S. J., Conejo A. J., Baringo L.. Investment in Electricity Generation and Transmission: Decision Making Under Uncertainty. Springer; 2016.

13. Shiina T., Birge J. R. Multistage stochastic programming model for electric power capacity expansion problem. Japan journal of industrial and applied mathematics. 2003;20(3):379-397.

14. Baringo L, Conejo A. J.. Wind power investment within a market environment. Applied Energy. 2011;88(9):3239-3247.

15. Birge J. R., Louveaux F.. Introduction to Stochastic Programming. Springer; 1997.

16. Zugno M., Jónsson T., Pinson Pierre. Trading wind energy on the basis of probabilistic forecasts both of wind generation and of market quantities. Wind Energy. 2013;16:909-926.

17. Bitar E. Y., Rajagopal R., Khargonekar P. P., Varaiya P.. Bringing wind energy to market. IEEE Transactions on Power Systems. 2012;27(3):12251235.

18. Dent C. J., Bialek J. W., Hobbs B. F.. Opportunity cost bidding by wind generators in forward markets: analytical results. IEEE Transactions on Power Systems. 2011;26(3):1600-1608.

19. Rockafellar R.T., Uryasev S.. Optimization of conditional value-at-risk. Journal of risk. 2000;2(3):21-42.

20. Weber C.. Adequate intraday market design to enable the integration of wind energy into the European power systems. Energy Policy. 2010;38(7):3155-3163.

21. Trading Appendix 2 / Clearing Appendix 2: Contract Specifications. : Nasdaq Oslo ASA and Nasdaq Clearing AB; 2016.

22. Pinson P., Chevallier C., Kariniotakis N.G.. Trading wind generation from short-term probabilistic forecasts.. IEEE Transactions on Power Systems. 2007;22(3):1148-1156.

23. Pinson P.. Wind Energy: Forecasting challenges for its operational management. Statistical Science. 2013;28(4):564-585.

24. Pinson P., Papaefthymiou G., Klockl B., Nielsen H. Aa., Madsen H.. From probabilistic forecasts to statistical scenarios of short-term wind power production. Wind Energy. 2009;12:51-62.

25. Pinson P., Girard R.. Evaluating the quality of scenarios of short-term wind power generation. Applied Energy. 2012;96:12-20. 
26. Hagspiel S., Papaemannouil A., Schmid M., Andersson G.. Copula-based modeling of stochastic wind power in Europe and implications for the Swiss power grid. Applied Energy. 2012;96:33-44.

27. Peljo J.. Futures Pricing in the Nordic Electricity Market. Master's thesisDept. of Finance, School of Business, Aalto UniversityFinland2013. 


$\begin{gathered}\text { Financial market: } \\ \text { Years, months, } \\ \text { weeks, days }\end{gathered}$
$\begin{gathered}\text { Day-ahead market: } \\ 36-12 \text { hours }\end{gathered}$ $\begin{gathered}\text { Real-time markets: } \\ \text { 1hour }-10 \text { minutes }\end{gathered}$

FIGURE 1 Timeline for a producer participating in multiple trading floors. 

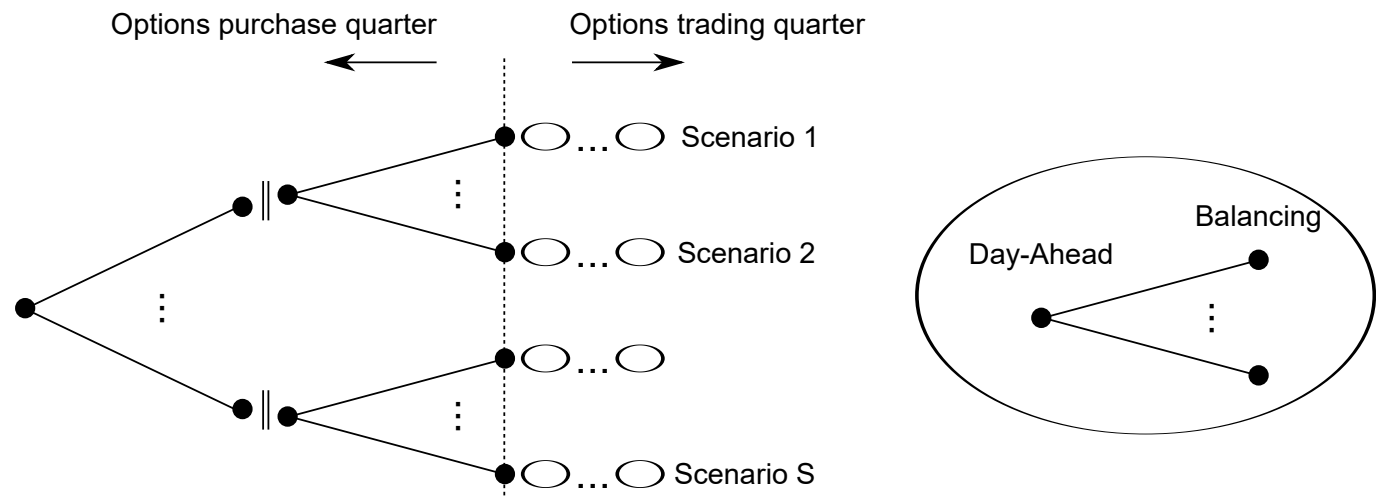

FIGURE 2 Schematic representation of the full scale multi-stage stochastic optimisation problem for wind power trading in financial, day-ahead and balancing markets. 


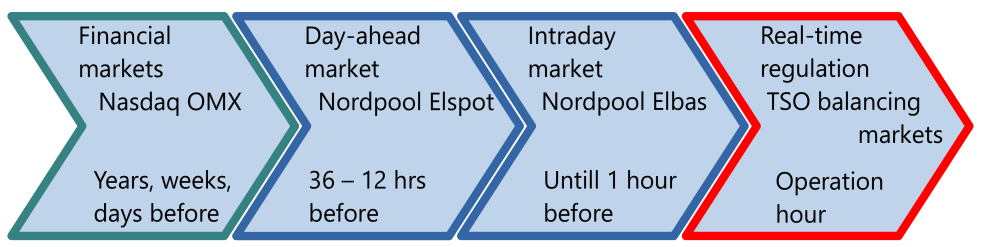

FIGURE 3 The structure of the Nordic electricity markets. 


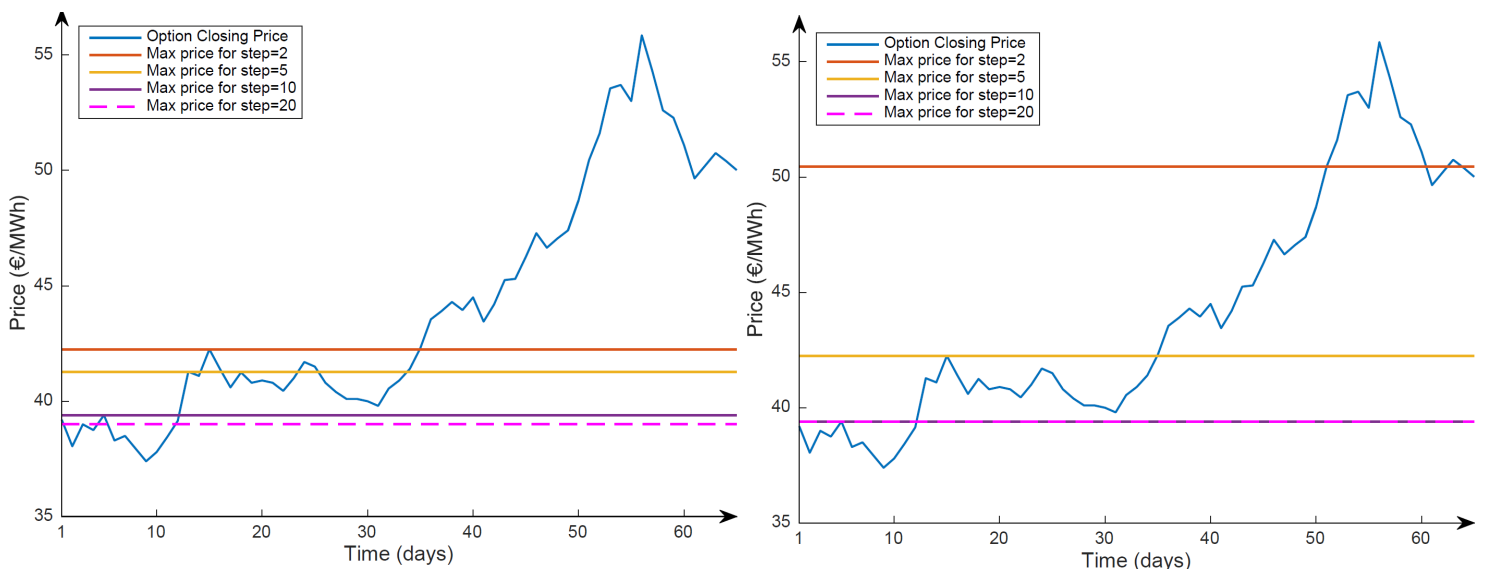

FIGURE 4 Option closing prices for quarterly total limits of 60 and 100 options and various purchase steps. 


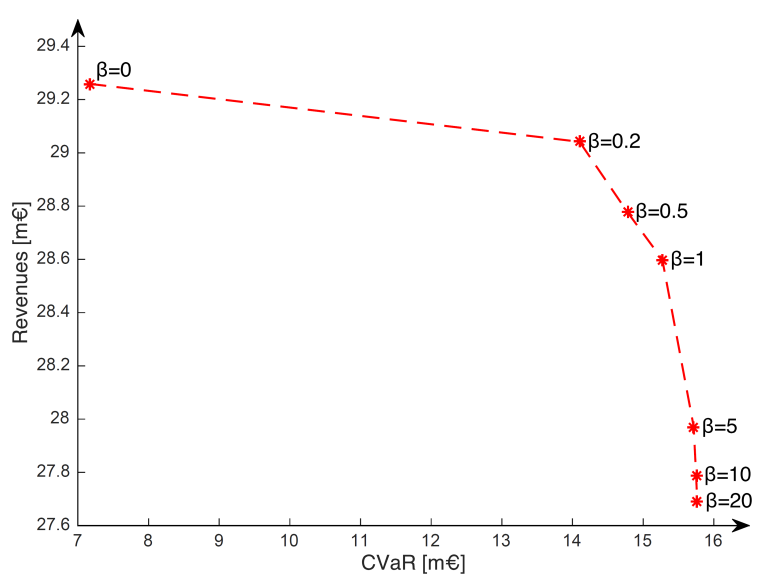

FIGURE 5 The impact of risk aversion parameter $\beta$ on total revenues. 
TABLE 1 Total profit for wind power producers.

\begin{tabular}{ccc}
\hline & Day-ahead market & Options and day-ahead market \\
\hline Deterministic model $[\mathrm{m} €]$ & 21.09 & 25.46 \\
Stochastic model $[\mathrm{m} €]$ & 23.39 & 26.89 \\
Perfect info. $[\mathrm{m} €]$ & 24.26 & 27.53 \\
\hline
\end{tabular}


TABLE 2 An overview of the combined trading strategy.

\begin{tabular}{cccc}
\hline & Deterministic model & Stochastic model & Perfect info. \\
\hline Total scheduled energy [GWh] & 650.59 & 727.86 & 676.88 \\
Energy sold in the day-ahead market [GWh] & 277.42 & 255.68 & 246.75 \\
Energy sold through options [GWh] & 373.17 & 472.18 & 430.13 \\
\hline Number of options exercised & 171 & 215 & 183 \\
\hline Positive imbalance $[\mathrm{MWh}]$ & 118.04 & 164.79 & - \\
Negative imbalance $[\mathrm{MWh}]$ & 91.76 & 215.77 & - \\
\hline Down-regulation costs $[\mathrm{m} €]$ & 3.56 & 5.50 & - \\
Up-regulation costs $[\mathrm{m} €]$ & 3.53 & 6.42 & - \\
Total revenue $[\mathrm{m} €]$ & 25.46 & 26.89 & 27.53 \\
\hline Performance ratio $(\gamma)[\%]$ & 92.48 & 97.68 & 100 \\
\hline
\end{tabular}


TABLE 3 Total revenues for various quarterly and daily purchase limits.

\begin{tabular}{cccc}
\hline Daily / Quarterly limits & 60 options & 100 options & 200 options \\
\hline 2 options & 27.56 & 28.71 & - \\
5 options & 26.89 & 26.46 & 24.12 \\
10 options & 26.84 & 25.69 & 22.71 \\
20 options & 26.74 & 25.67 & 21.81 \\
\hline
\end{tabular}


TABLE 4 Amount of energy traded in options and day-ahead market based on the parameter $\beta$.

\begin{tabular}{cccc}
\hline $\begin{array}{c}\text { Weighting factor } \\
\beta\end{array}$ & $\begin{array}{c}\text { Total traded energy } \\
\text { GWh }\end{array}$ & $\begin{array}{c}\text { Energy traded as options } \\
\text { GWh }\end{array}$ & $\begin{array}{c}\text { Energy traded in day-ahead market } \\
\text { GWh }\end{array}$ \\
\hline 0 & 727.86 & $472.18(64.9 \%)$ & $255.68(35.1 \%)$ \\
0.2 & 696.09 & $463.60(66.6 \%)$ & $232.49(33.4 \%)$ \\
0.5 & 666.54 & $455.90(68.4 \%)$ & $210.63(31.6 \%)$ \\
1 & 638.54 & $450.80(70.6 \%)$ & $187.74(29.4 \%)$ \\
5 & 573.40 & $425.75(74.2 \%)$ & $147.65(25.8 \%)$ \\
10 & 757.80 & $420.15(75.3 \%)$ & $137.64(24.7 \%)$ \\
20 & 549.02 & $416.84(75.9 \%)$ & $132.18(24.1 \%)$ \\
\hline
\end{tabular}

